\title{
Lifestyle interventions for mental health
}

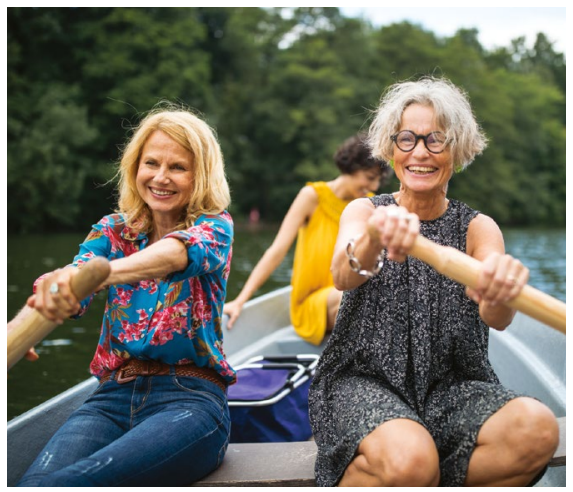

\section{CPD}

\section{Sam Manger}

\section{Background}

Mental illness is the single most common reason for patient visits to a general practitioner (GP). Prevalent mental illnesses include major depression disorder and dysthymia, anxiety disorders, bipolar disorders and schizophrenia. Patients with severe mental illness have a 10-20-year shorter life expectancy when compared with the general population, primarily due to physical chronic disease.

\section{Objective}

The aim of this article is to provide a concise update regarding the evidencebased clinical lifestyle interventions in the management of both mental illnesses and the physical chronic diseases highly prevalent in people with mental illness.

\section{Discussion}

Growing evidence shows that lifestyle interventions are an effective component of management for patients with mental illness to improve mental health, physical health and quality of life, which consequently assists in reducing the life expectancy gap between patients with severe mental illness and the general population. Measures can be taken to improve long-term adherence. The GP plays a crucial part in initiating and supporting patients with these measures.
ACCORDING TO THE 2018 General practice: Health of the nation report, mental health concerns are the single most common reason patients visit their general practitioners (GPs). ${ }^{1}$ The 12-month prevalence of anxiety disorders is $14.4 \%$, depressive disorders is $5.5 \%$, bipolar disorders is $1.8 \%$ and psychotic disorders (most commonly schizophrenia) is $0.45 \% .^{2}$ According to the World Health Organization, major depression disorder is the leading cause of disability in the world. ${ }^{3}$

Psychotherapeutic interventions (eg cognitive behavioural therapy) and medications have traditionally been the mainstays of treatment and can be effective in improving symptoms, functional outcomes and quality of life. ${ }^{4}$ However, the 2015 Royal Australian and New Zealand College of Psychiatrists' (RANZCP's) clinical practice guidelines for mood disorders have expanded the RANZCP model of care to include evidence-based lifestyle interventions as an important recommendation in addition to psychological therapies, medication and procedures. This model is termed the biopsychosocial lifestyle (BPSL) model. The BPSL model includes nutrition, physical activity, sleep, smoking cessation and reduction of substance abuse and alcohol consumption, all of which have been shown to affect the development and clinical management of depression and bipolar disorders. ${ }^{4}$
Furthermore, patients with severe mental illness have a 10-20-year life expectancy gap when compared with the general population; this gap is largely due to physical chronic disease, particularly cardiovascular and respiratory disease. ${ }^{5}$ In addition to the metabolic side effects of medication, mental illness is associated with potentially modifiable risk factors such as high rates of comorbid substance use, poor diet and physical inactivity, all of which contribute to poor physical health outcomes in this population. ${ }^{6}$ People with severe mental illness are 2-3 times more likely to have cardiovascular disease, respiratory illness, diabetes and osteoporosis, with up to $67 \%$ having metabolic syndrome. ${ }^{6,7}$

Lifestyle interventions can include a range of interventions that are beneficial for mental health, which at the same time improve the physical health variables that contribute to the life expectancy gap for patients with severe mental illness. The European Prospective Investigation into Cancer and Nutrition (EPIC) study found that improvements in lifestyle could prevent $93 \%$ of diabetes, $81 \%$ of heart attacks, $50 \%$ of strokes and $36 \%$ of all cancers. ${ }^{8}$ Furthermore, remission of multiple leading chronic diseases such as coronary artery disease and diabetes has been shown possible in numerous prospective trials. ${ }^{9,10}$ 


\section{Nutrition in mental illness}

Over the past 10 years there has been a marked increase in the amount of published literature showing evidence that higher-quality diets lead to a reduced risk for depression and lower-quality diets lead to an increased risk for depression. ${ }^{4,11}$ These studies of children, adolescents and adults have been published internationally. ${ }^{11}$ In 2017 , the randomised controlled 'Supporting the Modification of lifestyle in Lowered Emotional States' (SMILES) trial showed 32\% of patients with moderate-to-severe major depression disorder remit with nutritional interventions alone. ${ }^{12}$ In 2019, this was followed up with a large meta-analysis of $>45,000$ participants; the results showed that dietary interventions significantly reduced depression and anxiety symptoms, particularly in women. ${ }^{13}$

\section{Physical activity in mental illness In late 2018 the European Psychiatric Association issued new guidelines to promote physical activity as a fundamental management option for depressive disorders and even schizophrenia. ${ }^{14}$ For major depression disorder, consistent evidence indicates that physical activity can be as effective as antidepressants and psychotherapy in improving depression symptoms and quality of life, with greater effects shown when patients are supervised by an exercise specialist. Furthermore, for schizophrenia spectrum disorders, the guidelines indicate that physical activity can reduce psychiatric symptoms and improve cognition. ${ }^{14}$}

\section{Sleep in mental illness}

There is growing evidence of the role of sleep disorders in mental illness in a bidirectional manner, though this is a complex area of research. Studies have indicated the overall prevalence of sleep disorders in patients with a psychiatric illness is $40 \%$, with obstructive sleep apnoea, insomnia and restless leg syndrome being the most common. ${ }^{15}$ Studies have found that sleep disorders are a risk factor for new-onset and recurrent depression in children, adolescents and adults, affecting severity, duration and relapse rates. ${ }^{16}$ Between $30 \%$ and $80 \%$ of patients with schizophrenia have a sleep disorder, with poor sleep quality being associated with higher levels of symptom severity. ${ }^{17}$

\section{Smoking in mental illness}

Smoking rates are higher in people with mental illness, and the prevalence of smoking increases with the severity of the mental illness. Australian studies have found that approximately $70 \%$ of men and $60 \%$ of women diagnosed with psychotic disorders smoke. ${ }^{18}$ Smoking in people with mental disorders is an independent risk factor for suicide, with smoking cessation appearing to mitigate that risk. ${ }^{19}$ Higher doses of commonly prescribed psychiatric medications, such as olanzapine and clozapine, are required for people who smoke cigarettes because tobacco smoke induces cytochrome P450 enzymes in the liver, thereby affecting metabolism. Studies indicate that cessation of smoking can lead to a $30-50 \%$ reduction in the requirement of certain medication doses..$^{20}$ In 2014, a systematic review reported that people who give up smoking have a reduction in depressive and anxiety symptom severity and an improvement in mental health and quality of life, with the effect being equal to antidepressant therapy. ${ }^{21}$

\section{Improving adherence}

Adherence to lifestyle advice and medication varies significantly, with most studies showing an average of approximately $50 \% .{ }^{22}$ Poor adherence can be due to a variety of patient factors (eg perceived benefits, self-efficacy), clinician factors and healthcare system factors (eg time limits, cost, access). ${ }^{23}$ Adherence can be complicated for people with mental illness because of additional factors, including mental illness-related factors (eg paranoid symptoms, amotivation), side effects of medication (eg sedation), cognitive factors, psychosocial factors (eg social isolation, poverty) and clinician factors (eg stigma, lack of clinician training) ${ }^{24}$ Improving long-term adherence to treatment recommendations relies on addressing factors at multiple levels, including but not limited to: increased assessment, health coaching, telehealth, community engagement and involvement of a multidisciplinary team.

\section{Assessment}

Studies indicate that $22 \%$ of GPs usually assess nutrition, and $28 \%$ of GPs usually assess physical activity, with $38 \%$ rating their motivational skills as low and $52 \%$ as moderate. ${ }^{25,26}$ Cited barriers to assessment include time limitations, knowledge, confidence and funding. ${ }^{25}$ Training of GPs in lifestyle programs, such as the Australian Government's 'smoking, nutrition, alcohol and physical activity' (SNAP) program, can lead to a significant increase in assessment and management of lifestyle factors and increased behaviour change skills. ${ }^{26}$

\section{Health coaching}

The term 'health coaching' refers to a diverse set of behaviour change interventions, such as motivational interviewing and brief interventions, all with a shared aim of helping people set goals and take action. It may be facilitated by people with long-term conditions (peers), nurses, doctors or allied health professionals. There is evidence that health coaching can improve people's confidence and ability to self-manage their health conditions and assist them in adopting healthier lifestyle choices such as reducing smoking, eating more healthily and increasing physical activity. ${ }^{27}$ For people in rural locations or those who have other mobility barriers, programs such as telehealth can be an effective tool. Telehealth interventions produce positive outcomes when used for common chronic conditions, dietary interventions and behaviour change, with improvements in lifestyle factors, mortality and quality of life, and reductions in hospital admissions. ${ }^{28}$

\section{Community engagement}

Identifying high-risk populations for mental illness - such as adolescents, 

Aboriginal and Torres Strait Islander
peoples and carers of people with disabilities - and providing early lifestyle interventions is a key target and provides opportunities for partnerships with external programs and clinics. Community lifestyle programs such as 'Creating Opportunities for Personal Empowerment' (COPE) and mental health outreach programs such as 'I Got You: Healthy Life Choices for Teens' have shown that participants had improvements in healthy lifestyle behaviour, the ability to recognise mental health issues and high-risk behaviours, and their self-management. ${ }^{29}$

\section{Multidisciplinary approach}

Lifestyle approaches to mental health are both warranted and evidence-based at the clinical level. A multidisciplinary team may be required to comprehensively meet the medical and psychosocial needs of a patient living with mental illness, particularly when addressing lifestyle-related factors. The roots of many lifestyle, psychological and physical chronic diseases also lie in social risk factors such as isolation, lack of accommodation, financial distress, lack of education and occupation. ${ }^{30}$ Therefore, a multidisciplinary team is recommended and may consist of a GP, social worker, peer support person, case worker, psychologist, dietitian, exercise physiologist, addiction specialist, psychiatrist, diabetes educator and nurse.

\section{Conclusion}

Evidence-based and effective lifestyle therapies are indicated for people with mental illness in addition, or as an alternative, to usual care. Strong evidence shows that lifestyle interventions, such as nutrition, movement, sleep, stress management and substance cessation, are efficacious and cost-effective therapies that improve mental health, physical health and quality of life.

\section{Key points}

- Mental illness is the single most common reason for patient visits to a GP.
- Patients with severe mental illness have a 10-20-year life expectancy gap, primarily due to chronic physical disease.

- Evidence-based lifestyle interventions can be effective in the management of both mental illnesses and the physical chronic diseases highly prevalent in people with mental illness.

- Health coaching and increased lifestyle assessment may improve long-term adherence.

- A multidisciplinary team is required to comprehensively improve the lifestyle factors of a patient living with mental illness.

\section{Author}

Sam Manger MBBS, BSc, FRACGP, FASLM, General Practitioner, QId; President, The Australasian Society of Lifestyle Medicine (pro bono), Vic; Host, The GP Show Podcast (pro bono), Qld. sam@lifestylemedicine.org.au

Competing interests: $\mathrm{SM}$ is the pro bono president of The Australasian Society of Lifestyle Medicine. Funding: None.

Provenance and peer review: Commissioned, externally peer reviewed.

\section{References}

1. The Royal Australian College of General Practitioners. General practice: Health of the nation 2018. East Melbourne, Vic: RACGP, 2018.

2. Farrer LM, Walker J, Harrison C, Banfield M. Primary care access for mental illness in Australia: Patterns of access to general practice from 2006 to 2016. PLoS One 2018;13(6):e0198400 doi:10.1371/journal.pone.0198400.

3. World Health Organization. Depression and other common mental disorders: Global health estimates. Geneva: WHO, 2017.

4. Malhi GS, Bassett D, Boyce P, et al. Royal Australian and New Zealand College of Psychiatrists clinical practice guidelines for mood disorder. Aust N Z J Psychiatry 2015;49(1):1-185.

5. Lawrence D, Hancock K, Kisely S. The gap in life expectancy from preventable physical illness in psychiatric patients in Western Australia: Retrospective analysis of population based registers. BMJ 2013;346:f2539. doi: 10.1136/bmj.f2539.

6. Mental Health Commission of NSW. Physical health and mental wellbeing: An evidence guide. Sydney, NSW: Mental Health Commission of NSW, 2016

7. National Mental Health Commission. Equally well consensus statement: Improving the physical health and wellbeing of people living with mental illness in Australia. Sydney, NSW: NMHC, 2016.

8. Ford ES, Bergmann MM, Kröger J, Schienkiewitz A, Weikert C, Boeing $\mathrm{H}$. Healthy living is the best revenge: Findings from the European Prospective Investigation Into Cancer and Nutrition-Potsdam study. Arch Intern Med 2009;169(15):1355-62. doi: 10.1001/ archinternmed.2009.237.

9. Lean ME, Leslie WS, Barnes AC, et al. Primary care-led weight management for remission of type 2 diabetes (DiRECT): An open label, clusterrandomised trial. Lancet 2018;391(10120):541-51. doi: 10.1016/S0140-6736(17)33102-1.

10. Ornish D, Scherwitz L, Billings JH, et al. Intensive lifestyle changes for reversal of coronary heart disease. JAMA 1998;280(23):2001-07. doi: 10.1001/jama.280.23.2001.

11. O'Neil A, Quirk SE, Housden S, et al. Relationship between diet and mental health in children and adolescents: A systematic review. Am J Public Health 2014;104(10):e31-42. doi:10.2105/ AJPH.2014.302110.

12. Jacka FN, O'Neil A, Opie R, et al. A randomised controlled trial of dietary improvement for adults with major depression (the 'SMILES' trial). BMC Med 2017;15(1):23. doi: 10.1186/s12916-017-0791-y.

13. Firth J, Marx, W, Dash S, et al. The effects of dietary improvements on symptoms of depression and anxiety: A meta-analysis of randomised controlled trials. Psychosom Med 2019;81(3):265-80. doi: 10.1097/ PSY.0000000000000673.

14. Stubbs B, Vancampfort $D$, Hallgren $M$, et al. EPA guidance on physical activity as a treatment for severe mental illness: A meta-review of the evidence and position statement from the European Psychiatric Association (EPA), supported by the International Organization of Physical Therapists in Mental Health (IOPTMH). Eur Psychiatry 2018;54:124-44. doi: 10.1016/j. eurpsy.2018.07.004.

15. Hombali A, Seow E, Yuan Q, et al. Prevalence and correlates of sleep disorder symptoms in psychiatric disorders. Psychiatry Res 2018; pii: S0165-1781(18)30268-23. doi: 10.1016/j.psychres.2018.07.009.

16. Franzen PL, Buysse DJ. Sleep disturbances and depression: Risk relationships for subsequent depression and therapeutic implications. Dialogues Clin Neurosci 2008;10(4):473-81.

17. Kaskie RE, Graziano B, Ferrarelli F. Schizophrenia and sleep disorders: Links, risks, and management challenges. Nat Sci Sleep 2017;9:227-39. doi: 10.2147/NSS.S121076.

18. Mendelsohn CP, Kirby DP, Castle DJ. Smoking and mental illness. An update for psychiatrists. Australas Psychiatry 2015;23(1):37-43. doi: 10.1177/1039856214562076.

19. Prochaska JJ, Das S, Young-Wolff KC. Smoking, mental illness, and public health. Annu Rev Public Health 2017;38:165-85. doi: 10.1146/annurevpublhealth-031816-044618.

20. Tsuda Y, Saruwatari J, Yasui-Furukori N. Meta-analysis: The effects of smoking on the disposition of two commonly used antipsychotic agents, olanzapine and clozapine. BMJ Open 2014;4(3):e004216. doi: 10.1136/ bmjopen-2013-004216.

21. Taylor G, McNeill A, Girling A, Farley A, Lindson-Hawley N, Aveyard P. Change in mental health after smoking cessation: Systematic review and meta-analysis. BMJ 2014;348:g1151. doi: 10.1136/bmj.g1151.

22. Sabate E. Adherence to long-term therapies: Evidence for action. Geneva: WHO, 2003.

23. Devine F, Edwards T, Feldman SR. Barriers to treatment: Describing them from a different perspective. Patient Prefer Adherence 2018;12:129-33. doi: 10.2147/PPA.S147420.

24. The Royal Australian and New Zealand College of Psychiatrists. Keeping body and mind together: Improving the physical health and life expectancy of people with serious mental illness. Melbourne: RANZCP, 2015 
25. Denney-Wilson E, Fanaian M, Wan Q, Vagholkar S, Schütze $\mathrm{H}$, Harris M. Lifestyle risk factors in general practice - Routine assessment and management. Aust Fam Physician 2010;39(12):950-53.

26. Harris MF, Hobbs C, Powell Davies G, Simpson S, Bernard D, Stubbs A. Implementation of a SNAP intervention in two divisions of general practice: A feasibility study. Med J Aust 2005;183(10 Suppl):S54-58. doi: 10.5694/j.1326-5377.2005 tb07180.x.

27. Sforzo GA, Kaye MP, Todorova l, et al. Compendium of the health and wellness coaching literature. Am J Lifestyle Med 2017;12(6):436-47. doi 10.1177/1559827617708562.

28. Hanlon P, Daines L, Campbell C, McKinstry B, Weller D, Pinnock H. Telehealth interventions to support self-management of long-term conditions: A systematic metareview of diabetes, heart failure, asthma, chronic obstructive pulmonary disease, and cancer. J Med Internet Res 2017;19(5):e172. doi:10.2196/jmir.6688.
29. Melnyk BM, Jacobson D, Kelly S, et al. Promoting healthy lifestyles in high school adolescents: A randomized controlled trial. Am J Prev Med 2013;45(4):407-15. doi: 10.1016/j. amepre.2013.05.013.

30. World Health Organization and the Calouste Gulbenkian Foundation. Social determinants of mental health. Geneva: WHO, 2014. 